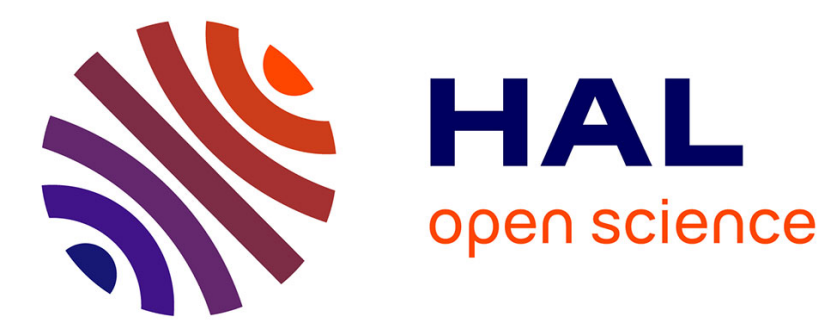

\title{
Villemaur-sur-Vanne "Les Orlets" (Aube), quatrième et dernière minière de l'autoroute A5. Résultats préliminaires
}

Pierre Arnaud Labriffe, Anne Augereau, Isabelle Sidéra, Frédéric Ferdouel

\section{- To cite this version:}

Pierre Arnaud Labriffe, Anne Augereau, Isabelle Sidéra, Frédéric Ferdouel. Villemaur-sur-Vanne "Les Orlets" (Aube), quatrième et dernière minière de l'autoroute A5. Résultats préliminaires. Revue archéologique de Picardie, 1995, 19ème colloque interrégional néolithique d'Amiens 1992., 9, pp.105119. 10.3406/pica.1995.1833 . halshs-02960556

\section{HAL Id: halshs-02960556 \\ https://shs.hal.science/halshs-02960556}

Submitted on 7 Oct 2020

HAL is a multi-disciplinary open access archive for the deposit and dissemination of scientific research documents, whether they are published or not. The documents may come from teaching and research institutions in France or abroad, or from public or private research centers.
L'archive ouverte pluridisciplinaire HAL, est destinée au dépôt et à la diffusion de documents scientifiques de niveau recherche, publiés ou non, émanant des établissements d'enseignement et de recherche français ou étrangers, des laboratoires publics ou privés. 


\title{
Villemaur-sur-Vanne «Les Orlets» (Aube), quatrième et dernière minière de l'autoroute A5. Résultats préliminaires \\ Pierre Arnaud Labriffe, Anne Augereau, Isabelle Sidéra, Frédéric Ferdouel
}

\section{Résumé}

La minière des "Orlets", à Villemaur-sur- Vanne (Aube), est située sur le versant opposé de celle fouillée sur la même commune en 1991 (Villemaur-sur-Vanne "Le Grand Bois Marot") et présentée au colloque sur le Néolithique à Dijon ainsi qu'à la Table ronde de Vesoul sur les "Mines de silex au Néolithique en Europe occidentale". Egalement menacée par la construction de l'autoroute A5, ce site, dont la superficie totale est estimée entre 25 et 40 ha, elle a fait l'objet d'une fouille de sauvetage programmé sur une superficie d'environ un hectare. La phase terrain s'est déroulée sur quatre mois entre février et mai 1992 ; les études sont encore en cours et ce ne sont que des résultats préliminaires qui sont présentés ici.

\begin{abstract}
The "Orlets" surface mine of Villemaur-sur- Vanne (Aube) is on the hillside opposite the mine already searched on the same territory in 1991 (Villemaur-sur- Vanne "Le Grand Bois Marot") and discussed at the Dijon Neolithic symposium and at the Vesoul Round Table on "Neolithic silex mines in Western Europe". The site which covers possibly between 25 and 40 hectares, has been sujected to emergency safeguard excavating over approximately one hectare during four months from February to May 1992. The study is still in progress and only prliminary results are given here.
\end{abstract}

\section{Citer ce document / Cite this document :}

Labriffe Pierre Arnaud, Augereau Anne, Sidéra Isabelle, Ferdouel Frédéric. Villemaur-sur-Vanne «Les Orlets» (Aube), quatrième et dernière minière de l'autoroute A5. Résultats préliminaires. In: Revue archéologique de Picardie. Numéro spécial 9, 1995. 19ème colloque interrégional néolithique d'Amiens 1992. pp. 105-119;

doi : https://doi.org/10.3406/pica.1995.1833

https://www.persee.fr/doc/pica_1272-6117_1995_hos_9_1_1833

Fichier pdf généré le 10/09/2020 


\title{
VILLEMAUR-SUR-VANNE “LES ORLETS" (AUBE) QUATRIEME ET DERNIERE MINIERE DE L'AUTOROUTE A5. RESULTATS PRELIMINAIRES
}

\author{
Pierre-Arnaud de LABRIFFE*, Anne AUGEREAU**, Isabelle SIDERA**, Frédéric FERDOUEL***.
}

\begin{abstract}
Résumé
La minière des "Orlets", à Villemaur-sur-Vanne (Aube), est située sur le versant opposé de celle fouillée sur la même commune en 1991 (Villemaur-sur-Vanne "Le Grand Bois Marot") et présentée au colloque sur le Néolithique à Dijon ainsi qu'à la Table ronde de Vesoul sur les "Mines de silex au Néolithique en Europe occidentale". Egalement menacée par la construction de l'autoroute A5, ce site, dont la superficie totale est estimée entre 25 et $40 \mathrm{ha}$, elle a fait l'objet d'une fouille de sauvetage programmé sur une superficie d'environ un hectare. La phase terrain s'est déroulée sur quatre mois entre février et mai 1992 ; les études sont encore en cours et ce ne sont que des résultats préliminaires qui sont présentés ici.
\end{abstract}

\begin{abstract}
The "Orlets" surface mine of Villemaur-sur-Vanne (Aube) is on the hillside opposite the mine already searched on the same territory in 1991 (Villemaur-sur-Vanne "Le Grand Bois Marot") and discussed at the Dijon Neolithic symposium and at the Vesoul Round Table on "Neolithic silex mines in Western Europe". The site which covers possibly between 25 and 40 hectares, has been sujected to emergency safeguard excavating over approximately one hectare during four months from February to May 1992. The study is still in progress and only prliminary results are given here.
\end{abstract}

\section{LOCALISATION DU SITE}

Le tracé de l'autoroute A5 qui reliera Melun à Troyes via Sens s'est avéré particulièrement propice à la découverte, la fouille et l'étude de sites d'extraction. En effet quatre gisements de ce type ont pu y être fouillés. Serbonnes "Le Revers de Brossard" (Yonne), situé sur la rive gauche de la vallée de l'Yonne (MENDOZA Y ALMEIDA M. et HASCOET J., 1990 ; HASCOET J. et al., 1993 ; SIDERA I., 1991 a), et dans l'Aube trois sites répartis sur moins de deux kilomètres de tracé ont également pu y être fouillés. Il s'agit de Pâlis "Le Buisson Gendre" (HASCOET J. et MENDOZA Y ALMEIDA M., 1991 b et $\mathrm{c}$ ), et des deux minières de Villemaur (fig. 1).

La minière des "Orlets" à Villemaur-sur-Vanne est située à une vingtaine de kilomètres à l'ouest de Troyes et une quarantaine de Sens (fig. 1). Dans ce secteur une dizaine de sites d'extraction sont actuellement repérés (fig. 2). Nous les avons regroupés sous la notion de complexe minier (LABRIFFE P.-A. de et THEBAULT D., 1991 a, et sous presse). Le site des "Orlets", composante de cet ensemble, est localisé sur le versant oriental $\mathrm{d}^{\prime}$ une petite vallée sèche qui débouche sur la rive droite de la Vanne. Sur le versant occidental de cette vallée se trouve le gisement du Grand Bois Marot (fig. 3). Cette minière dont une partie a également été fouillée dans le cadre des fouilles de sauvetage de l'autoroute $\mathrm{A} 5$ a été présentée à l'occasion du colloque de Dijon (AUGEREAU A., 1991 c ; LABRIFFE P.-A. de, THEBAULT D., 1991 b) et de la table ronde de Vesoul (AUGEREAU

* S.R.A. de Champagne-Ardenne; 20, rue de Chastillon F - 51000 CHALONS-SUR-MARNE.

** E.R.A. 12 du C.N.R.S 3, rue Michelet,

F - 75006 PARIS.

*** A.F.A.N.; 10, rue Pierre Brossolette, F - 10160 AIX-EN-OTHE

\section{REMERCIEMENTS}

Nous tenons à exprimer nos remerciements à J. Pelegrin, de L'ERA 28 du CNRS, pour avoir réalisé la taille expérimentale et pour tout l'intérêt qu'il porte à nos travaux.

$\dot{A}$. Augereau s'est plus particulièrement occupée des aspects touchant la production lithique; l'étude de l'outillage d'extraction en bois de cerf a été confié à I. Sidéra assistée de F. Ferdouel. 


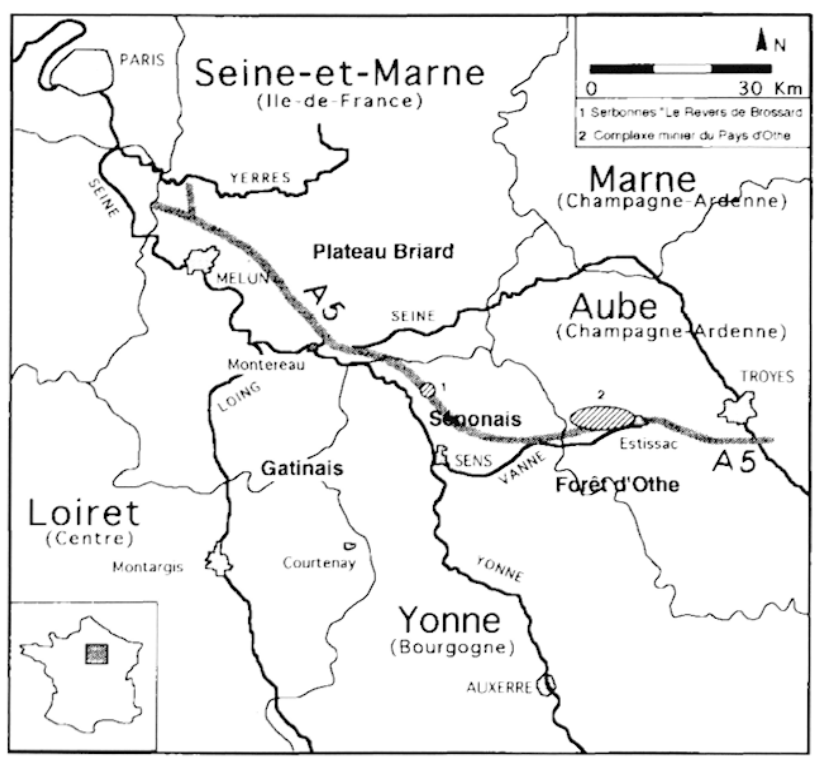

Fig. 1 : emplacement des mines de silex fouillées sur le tracé de l'Autoroute A5.

A., sous presse ; LABRIFFE P.-A. de et THEBAULT D., sous presse ; SIDERA I., sous presse). Ces deux sites localisés en vis-à-vis sont dans le même contexte géologique, au coeur du Sénonais.

Le Sénonais est un pays de collines crayeuses entourées par les grandes vallées alluviales de la Seine et de l'Yonne. Ces collines sont parfois encore surmontées de résidus tertiaires qui contiennent de nombreux grès erratiques propices au polissage. $D^{\prime}$ ailleurs le département de l'Aube était au début du siècle le département de France dans lequel le plus de polissoirs avaient été répertoriés. La plupart d'entre eux sont localisés dans le Pays d'Othe à proximité des zones d'extraction (JOURDAIN D., 1991)

\section{CIRCONSTANCES DE LA DECOUVERTE}

Dès les phases préliminaires de prospection sur le tracé, au printemps 1989, à partir d'informations fournies par R. Dijon, amateur qui a prospecté plus de cinquante ans dans la région, nous pouvions observer une série de structures d'extraction à trois cents mètres au nord de l'emprise, dans le talus fraîchement réavivé d'un chemin agricole. Quelques mois plus tard à l'occasion d'un survol du tracé par la Coordination Archéologique A5, J.L. Rieu photographiait des anomalies pouvant correspondre à des structures d'extraction. Mais là encore à quelques centaines de mètres du tracé.

Sur le passage de l'emprise aucun indice particulier n'avait été repéré, bien que la parcelle concernée ait fait l'objet d'une prospection de surface, mais dans de mauvaises conditions. Par contre, l'ensemble du versant était connu pour recéler un nombre impressionnant de haches. La collection de R. Dijon par exemple contient plus de 600 ébauches provenant de ce secteur. La renommée de ce site a d'ailleurs largement dépassé les limites de la région puisque nous y avons même rencontré un prospecteur savoyard.

$\mathrm{D}^{\prime}$ après les informations que nous avions recueillies, les zones les plus favorables aux ramassages étaient situées à quelques centaines de mètres plus au nord ou plus au sud de l'emprise. Tous les prospecteurs de la région nous avaient dit qu'à l'endroit de l'emprise elle-même il ne fallait pas s'attendre à trouver quoi que ce soit. Il semblait y avoir un hiatus au niveau de l'autoroute que l'existence d'un léger talweg aurait pu justifier (fig.3).

Fig. 2 : les sites du complexe minier du Pays d'Othe.

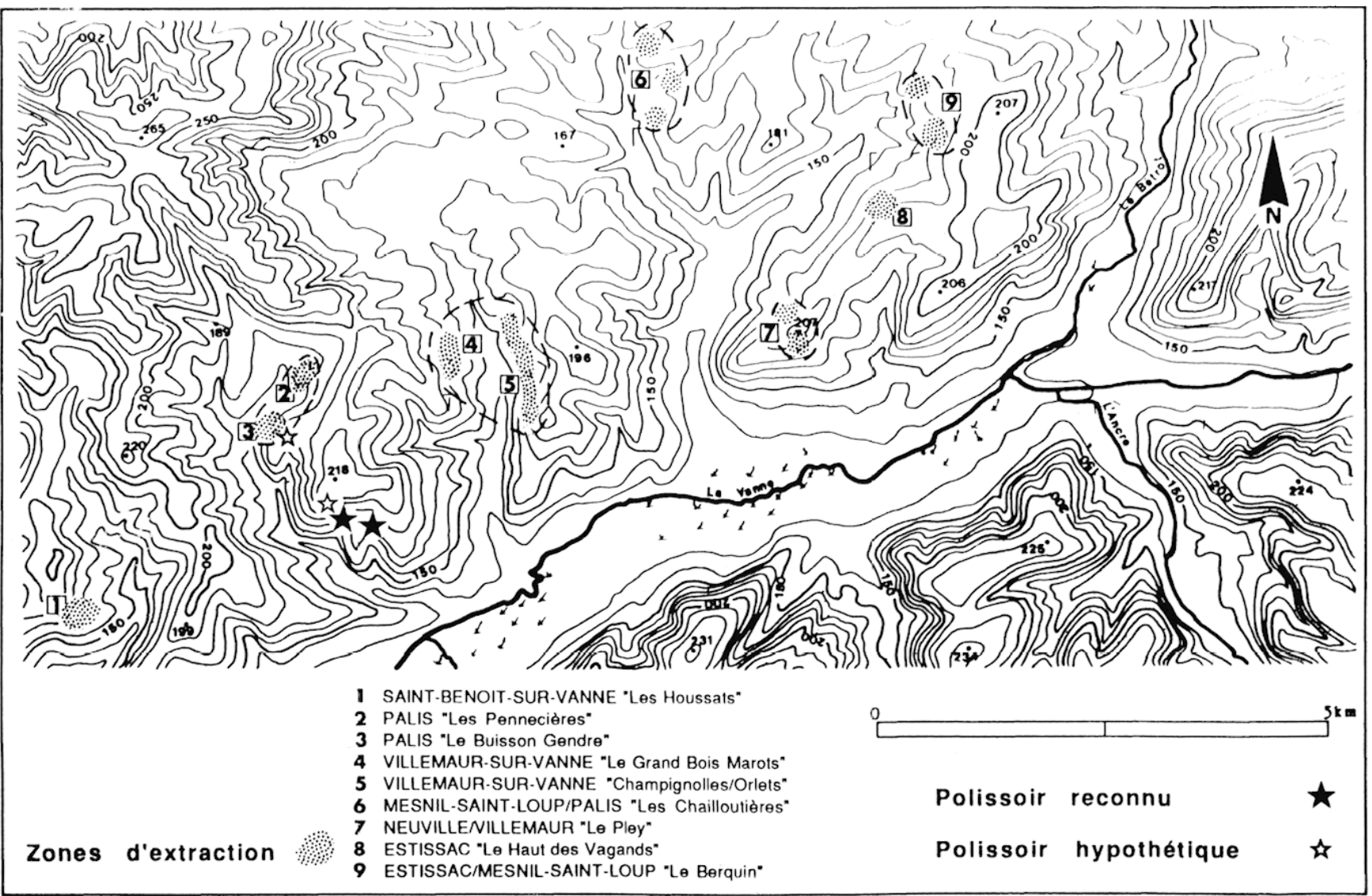




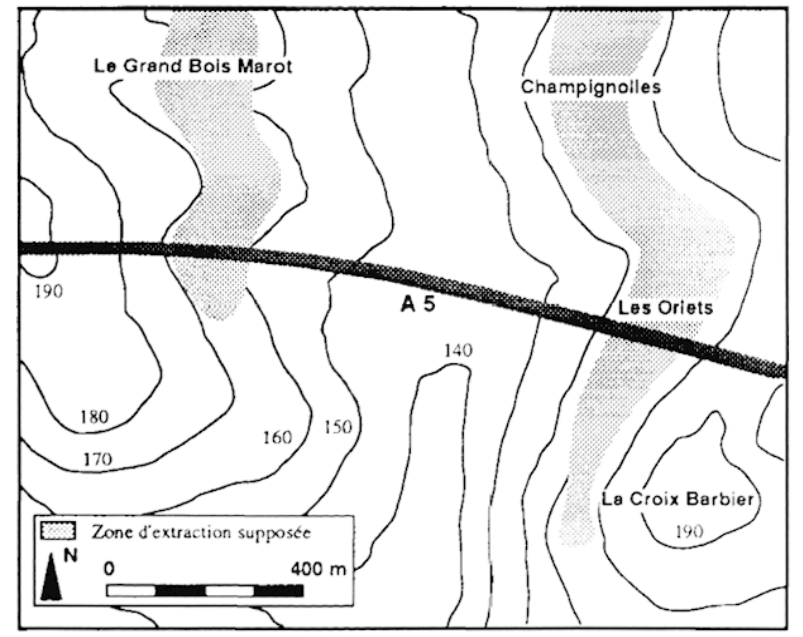

Fig. 3 : localisation des minières du Grand Bois Marot et des Orlets.

Malgré ces échos plutôt négatifs, et après les moissons, des tranchées de diagnostic étaient effectuées de part et d'autre de l'emprise dans le courant de l'été 91 (fig.4). Sur la parcelle des Orlets, un nombre particulièrement important de structures d'extraction étaient rapidement mises au jour. Un rapide diagnostic était réalisé, consistant en un relevé en plan des structures apparues et un relevé de quelques stratigraphies obtenues après sondage à la pelle mécanique dans une demi-douzaine de puits ou fosses.

Les objectifs de cette intervention étaient de mettre en évidence les principales caractéristiques de ce site et de définir une stratégie d'intervention. Les résultats étaient exposés dans un rapport servant de support à une demande de sauvetage program- mé (LABRIFFE P.-A. de, 1992).

Les principaux enseignements que nous tirions de l'opération de diagnostic étaient le suivants : absence de formations superficielles, impression d'extrême densité des structures, ateliers de taille peu ou pas conservés, grande variabilité des structures d'extraction, outillage d'extraction en bois de cerf, production lithique avec quelques éléments laminaires. Le schéma d'intervention proposé était très comparable à ceux déjà réalisés sur les précédentes minières.

\section{DEROULEMENT DES OPÉRATIONS DE TER- RAIN}

Pour des raisons de météo, de plannings et de disponibilité des équipes, la fouille proprement dite n'a pu commencer avant la fin de l'hiver 91-92. Les terrassements autoroutiers devant commencer au début du mois de juin, la fouille s'est déroulée sur quatre mois entre février et mai 1992, avec l'aide d'une équipe de quatre à dix personnes.

L'intégralité de la surface concernée a été décapée soit une superficie d'environ $12000 \mathrm{~m} 2$ (fig. 4). Tout le mobilier situé dans le labour ou au sommet des structures d'extraction a été prélevé et un relevé en plan de l'ensemble des structures a été effectué. Puis nous avons réalisé deux tranchées dans l'axe de l'autoroute, larges d'à peu près $6 \mathrm{~m}$ et dont la profondeur variait en fonction de celle des puits (fig. 4). Par la suite nous avons procédé au relevé des stratigraphies des structures d'extraction qui avaient été recoupées, et fouillé manuellement les

Fig. 4 : plan du secteur fouillé.

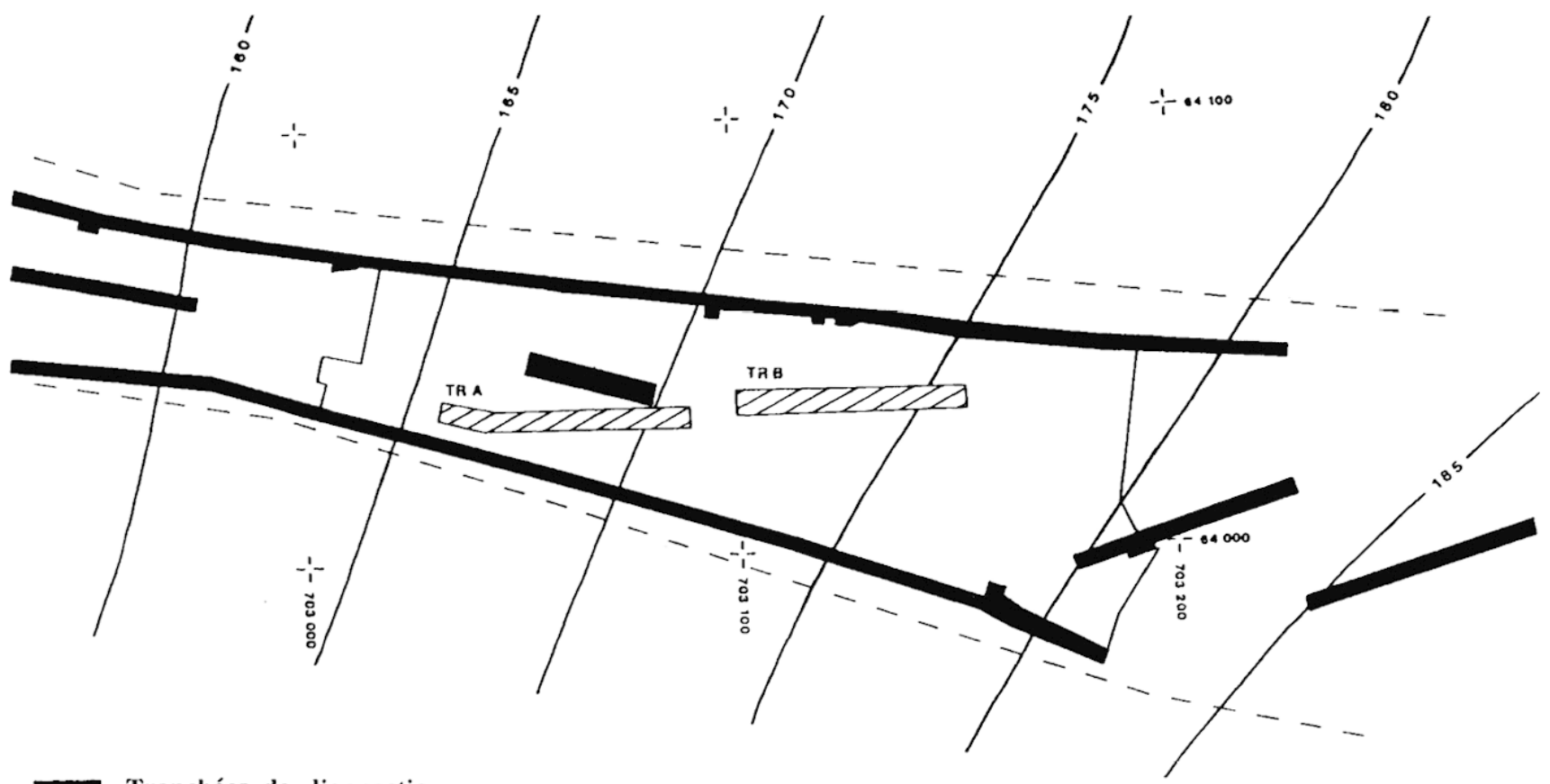

\footnotetext{
¿2 Tranchées profondes

$\square$ décapage
}

_ _ Linite d'emprise

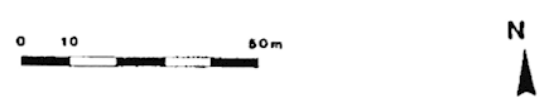


portions restantes des puits.

L'équipe de géomètres-topographes de la Coordination Archéologique A5 a effectué des relevés en trois dimensions de certains puits, à partir des méthodes mises au point sur la minière de Jablines (LAPROTE L., 1992). Ce type de relevé est le meilleur moyen pour rendre compte de la complexité des structures. Une portion de chambre d'extraction ainsi que de traces d'outils réparties dans plusieurs structures d'extraction ont été moulées dans une perspective de reconstitution au Musée de Sens (1).

Sur une période de quatre mois, ce sont en tout plus de soixante structures d'extraction dont les stratigraphies ont été relevées et quarante d'entre elles ont été fouillées manuellement. La volonté que nous avions de caractériser un ensemble plutôt que quelques éléments isolés, dans un temps relativement limité, nous a conduit à effectuer des choix draconiens.

\section{ASPECTS GEOLOGIQUES}

Contrairement aux "Bois Marots" il n'y a pas de formations superficielles sur ce site. La craie affleure immédiatement sous une fine couche de terre végétale (une trentaine de centimètres). Ce phénomène est caractéristique des dissymétries de versant que l'on rencontre dans ce pays de collines crayeuses. Bien qu'un léger doute subsiste, il semble bien que les sites d'extraction aubois se soient implantés sur du Coniacien supérieur.

Quatre bancs de silex ont été exploités. Ils sont globalement horizontaux et autant qu'on puisse le deviner par les négatifs de rognons laissés dans les parois des puits, ils devaient être réguliers. Leur disposition, combinée à la forte pente qui existe, les fait tous affleurer sur le versant à un moment ou un autre (fig. 5).
Les bancs 1 et 2 (bancs supérieurs) n'ont été que faiblement exploités. Ils sont discontinus et constitués de petits rognons présentant parfois des excroissances cornues.

Les bancs 3 et 4 ont subi une exploitation particulièrement poussée. A certains endroits il ne reste quasiment plus de matière première en place. Le banc 3 se présente sous la forme d'une dalle continue épaisse de 10 à $15 \mathrm{~cm}$ mais que la microtechtonique a par endroits totalement faillée.

Le banc 4 est dédoublé et n'est pas continu. Il est constitué de rognons de taille et de forme variées. On pourrait même parler de deux bancs puisqu'il y a deux lits de silex espacés d'une vingtaine de centimètres l'un de l'autre.

Les conditions gitologiques s'avèrent particulièrement favorables et sont vraisemblablement en grande partie responsables de l'implantation du site et de son exploitation intensive.

\section{ORGANISATION EN PLAN DE LA MINIERE}

Comme sur les deux autres gisements de ce type que nous avions déjà étudiés dans l'Aube les travaux sont passés perpendiculairement au développement de l'exploitation (fig. 3). Nous avons pu cerner les limites est et ouest du site. Mais comme le laissaient penser les prospections de surface, la mine se développe largement au nord et au sud de part et d'autre de l'emprise (fig. 6).

Sur le secteur décapé, l'anthropisation du sous-sol atteint des proportions inégalées sur les autres sites d'extraction et rend le plan particulièrement complexe à déchiffrer. Ce phénomène est particulièrement marqué dans le tiers central du décapage

(1) - Le moulage a été réalisé en résine par $\mathrm{S}$. Fourquet et M. Delaune.
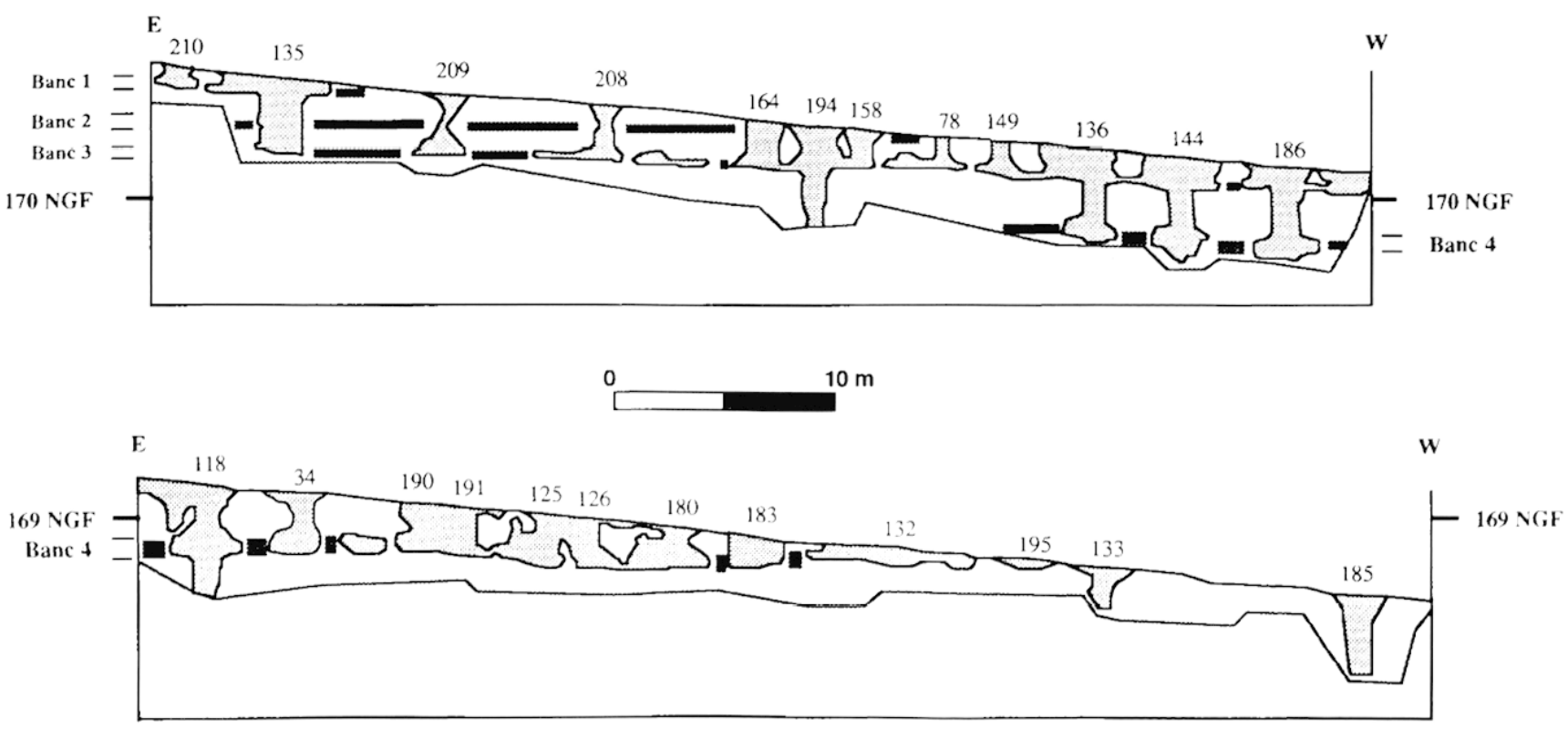

Fig. 5 : relations entre structures d'extraction et substrat; relevés des tranchées $A$ et $B$, coupe sud. 
où il y a de très nombreux recoupements de structures qui finissent par former un entremêlement inextricable et difficilement interprétable (Fig. 6).

Le dénombrement exact des puits découverts est pratiquement impossible. Nos estimations laissent penser qu'il y aurait eu environ un millier de puits sur ce secteur. Toutefois, cette estimation est à pondérer par le fait qu'aux "Orlets" elle a été réalisée à partir du dénombrement effectué dans les tranchées de diagnostic et que ces dernières sont de petite taille. Malgré tout, après le décapage intégral de la surface, l'impression d'extrême densité pressentie au moment du diagnostic s'est avérée largement fondée. Si on compare la minière des "Orlets" aux trois autres gisements fouillés sur l'A5 ou à Jablines, ce site semble avoir subi l'exploitation la plus intensive (tab. I).

\begin{tabular}{lccc}
\hline & $\begin{array}{c}\text { Nb } \\
\text { puits }\end{array}$ & $\begin{array}{c}\text { Zone d'extraction } \\
\text { décapeé }\end{array}$ & $\begin{array}{c}\text { Densité } \\
\text { / Ha }\end{array}$ \\
\hline JABLINES & 766 & 29300 & 261,4 \\
SERBONNES & 95 & 2529 & 375,6 \\
PALIS & 120 & 3600 & 333,3 \\
BOIS MAROT & 90 & 2515 & 357,9 \\
ORLETS & 107 & $\mathbf{9 5 2}$ & $\mathbf{1 1 2 4}$ \\
\hline
\end{tabular}

Il est encore difficile de conclure si la densité exceptionnelle rencontrée aux "Orlets" est représentative de l'ensemble de la minière ou si nous sommes sur un secteur d'exploitation tout à fait exceptionnel. Malheureusement nous ne disposons que d'informations lacunaires sur l'organisation du site en dehors de la zone fouillée. En effet, les quelques clichés aériens dont nous disposons ne font pas apparaître un semis de puits, contrairement à Jablines.

\section{ACQUISITION DE LA MATIERE PREMIERE}

La morphologie des structures d'extraction est variée et largement conditionnée par la gitologie. Les profondeurs sont comprises entre $30 \mathrm{~cm}$ et $5,80 \mathrm{~m}$.

A l'est du site les structures d'extraction présentent un tissu relativement lâche. Le banc 1 affleure et a fait l'objet d'unc récupération limitée au moyen de petites fosses qui possèdent des chambres d'extrac-

Tableau I : densité de l'occupation sur différents sites d'extraction (tableau et graphique).

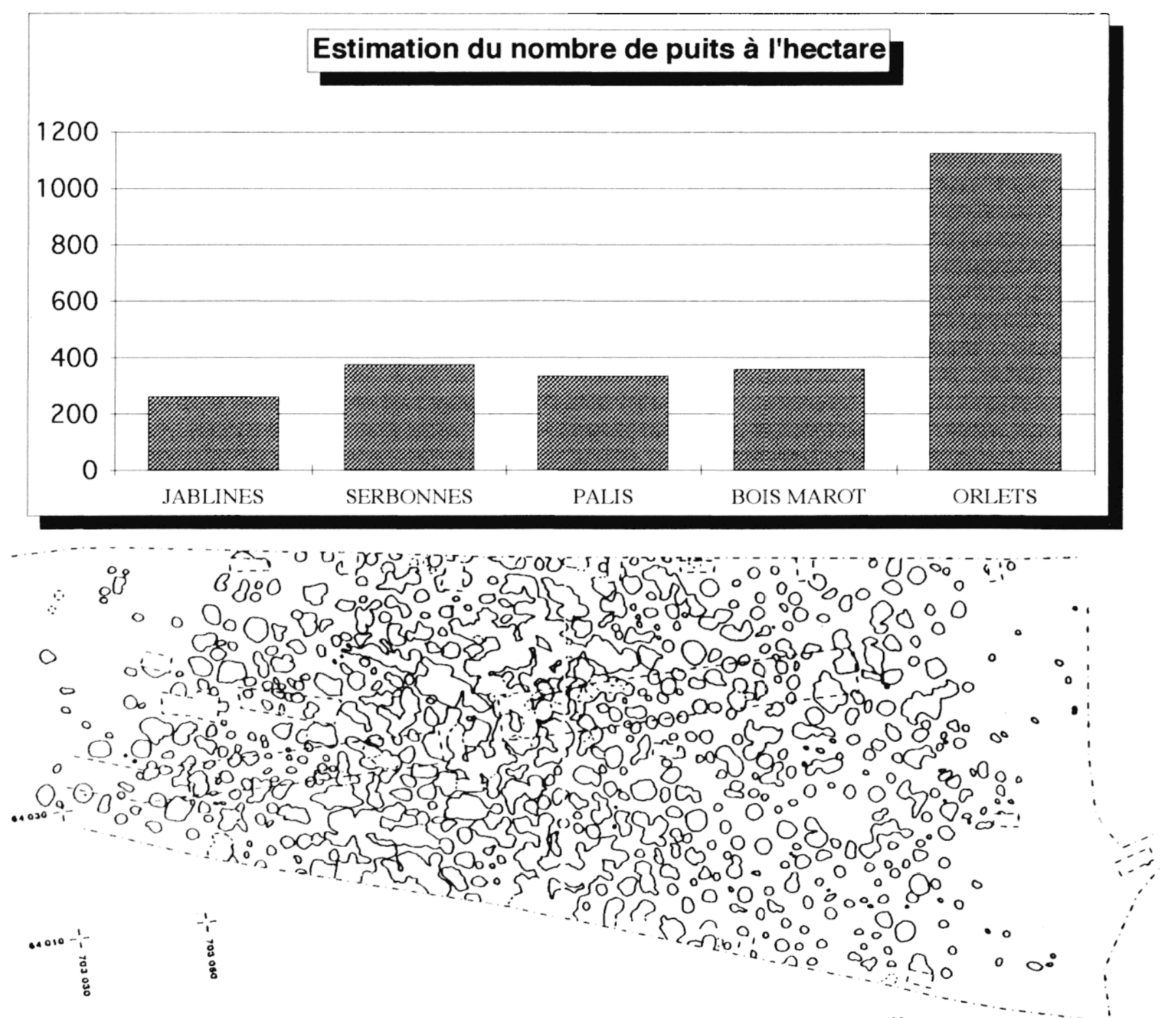

Fig. 6. : plan de la minière. 
Fig. 7 : coupe stratigraphique de la St. 135. Mise au net Y. Amrane, S. Verneau.

tion de taille parfois extrêmement réduite qui pourrait faire penser à du travail d'enfants (fig. 5 , tranchée B).

Le banc 2 n'a quasiment pas été exploité en tant que tel. Il a le plus souvent été extrait par raccroc par des puits exploitant également les bacs 3 ou 4 (fig. 5 tranchée B et fig. 7).

Le banc 3 a totalement été exploité. La faible profondeur d'enfouissement combinée à la régularité de sa disposition l'ont rendu particulièrement attractif pour les populations néolithiques (fig.5, tranchée B). A l'est il l'a été par des puits que l'on pourrait qualifier de "classiques". Ils possèdent une courte cheminée qui s'ouvre sur des chambres d'extraction. Au centre de la minière (fig. 5, tranchée $B$ ), ce banc a fait l'objet d'une récupération frénétique. On constate une anthropisation presque totale du sol et du sous-sol. Ceci rend relativement difficile une bonne caractérisatrion des structures d'extraction.

Dans le même secteur et à espacements à peu près réguliers, il y a une série de puits profonds qui ont exploité conjointement les bancs 3 et 4 (Fig. 5 , tranchée $B$, st. 136, 144, 186 et fig. 8). Ces puits à deux niveaux sont des structures impressionnantes. Le banc 4 est à environ 3,5 mètres de la surface du sol, mais dans la mesure où ils ont toujours été légèrement surcreusés, ils font plus de 4 mètres de haut. Ces puits n'étaient pas clairement discernables en surface car ils sont situés dans la zone surexploitée rencontre le niveau d'exploitation du banc 3 . Celuici se présente sous la forme d'une série de chambres dont la forme originelle est difficilement perceptible car l'exploitation ayant été intensive, elles ont toutes été reprises à un moment ou un autre par différents creusements.

Sur le radier de ce niveau, à peu près au centre, il y a un conduit d'un peu plus de $2 \mathrm{~m}$ de haut, parfaitement cylindrique ( $1 \mathrm{~m}$ de diamètre), qui permet d'accéder au banc 4 . Ce banc est exploité par un élargissement du conduit sous la forme de chambres d'extraction (2). Elles sont relativement haute pour deux raisons : $d^{\prime}$ une part elles ont souvent été l'objet d'effondrements des parois postérieurs à l'abandon des puits, d'autre part le banc 4 est dédoublé et les rognons sont répartis sur une épaisseur de plus de 40 centimètres.

Nous avons pu remarquer une certaine tendance de ces puits à posséder un surcreusement au centre, à l'aplomb du conduit d'accès entre les bancs 3 et 4 . Nous avons également constaté qu'il y avait fréquemment dans les stratigraphies des traces de pieux en bois semblant avoir été posés sur le fond des puits. Dans ces cas précis nous ne savons pas encore si les surcreusements sont à mettre en relation avec une technique particulière de creusement des puits ou s'ils n'ont servi qu'à la mise en place de systèmes de levage ou d'échelles.

(2) - C'est l'une d'entre elle qui a fait l'objet d'un moulage. 
Fig. 8 : coupe stratigraphique de la St. 144. Mise au net $Y$. Amrane, S. Verneau.

A l'ouest (fig. 5, tranchée A), le banc 4 de moins en moins profond a, comme le banc 3 , été totalement prélevé. La profondeur et la morphologie des structures d'extraction sont bien évidemment complètement liées à celles de la matière première. Plus on va vers l'ouest, plus les cheminées se réduisent pour aboutir à une exploitation en simple fosse sur le bas de la pente.

Sur la marge occidentale de la minière, là où l'extraction s'est faite en fosse, nous avons rencontré plusieurs cas de puits test (fig. 5, tranchée $A$, st. 185 et fig. 9). Ils sont profonds étroits, et n'ont semble-t-il servi qu'à reconnaître la nature du sous-sol et son potentiel en matière siliceuse. Ils ont été abandonnés sans avoir donné lieu à aucune exploitation, dans la mesure où ils n'ont rencontré aucun banc exploitable. Il est intéressant de noter que ces puits sont situés exactement au même endroit (bas de pente, marge de la minière) que celui qui avait été retrouvé sur le versant opposé, sur le site du "Grand Bois Marot".

Fig. 9 : coupe stratigraphique de la St. 185. Mise au net N. Bourgarel.

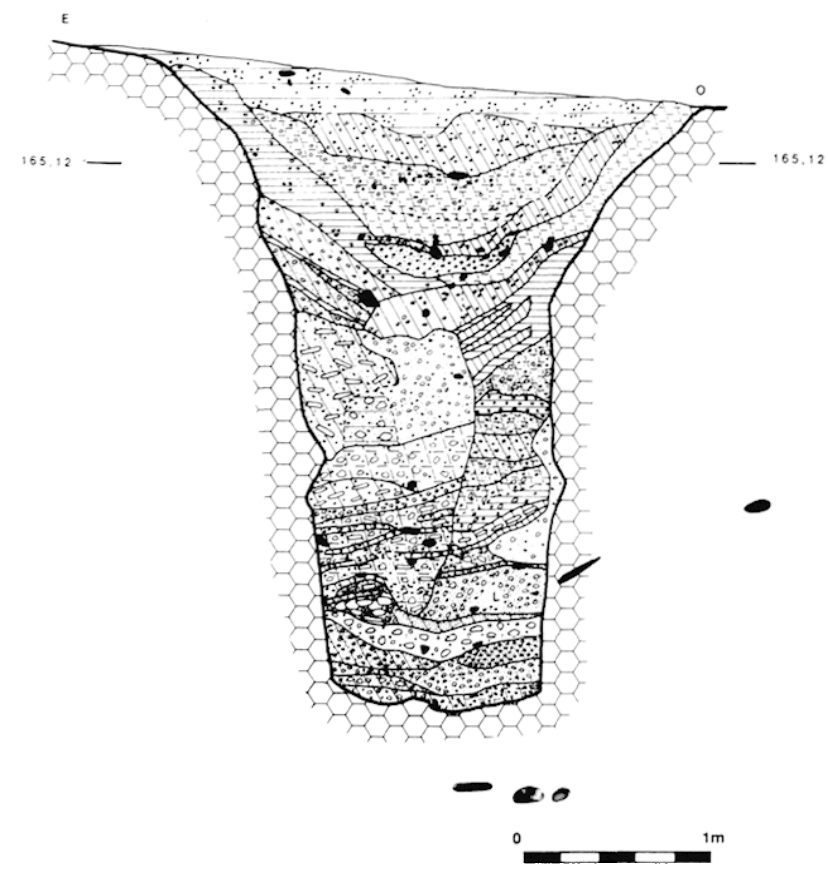


Bien que toutes les données n'aient pas encore été analysées, on peut dégager quelques régularités dans le système d'extraction. De manière systématique, les stériles sont dans la mesure du possible laissés dans les puits. Dès qu'un espace existe, celui-ci est colmaté par des remblais constitués de blocs ou dalles de craie provenant des chambres contigües en cours d'exploitation. Ceci est vérifié par la propreté et l'absence d'altération que présente la craie rencontrée dans les niveaux inférieurs des remplissages. Si ce matériau était resté un tant soit peu à l'air libre, en surface, il aurait immanquablement présenté des traces de ce séjour.

Cependant, les mineurs ont forcément dû remonter une partie des stériles. Ces déblais ont dû faire l'objet d'une politique de gestion organisée. En effet l'extrême complexité du plan en surface laisse penser que les morts terrains remontés ne devaient et ne pouvaient rester longtemps à l'air libre. Le volume de déblais produits aurait rapidement empêché de réaliser une exploitation aussi intensive que celle qui a été pratiquée. Cette constatation est corroborée par le fait qu'il y a dans plusieurs structures des couches dont le pendage est opposé au sens naturel de la pente. Celui-ci étant bien marqué il n'est pas possible d'envisager une mise en place naturelle, mais plutôt un remplissage volontaire avec des sédiments provenant de structures d'extraction en cours d'exploitation situées en contrebas et dans un environnement proche des premières.

Toutefois, le remplissage de la partie supérieure des puits est variable et il est vraisemblable qu' une partie des comblements sommitaux puisse être $\mathrm{d}^{\prime}$ origine naturelle. On y retrouve souvent une alternance de couche de craie et de limons. Parmi ces couches, il peut y avoir des concentrations de restes de débitage pouvant correspondre à l'érosion et au piégeage des ateliers de taille.

\section{L'OUTILAGE D'EXTRACTION}

La très grande majorité des outils d'extraction qui sont parvenus jusqu'à nous sont en bois de cerf. Mais ce n'est sans doute pas le seul matériau qui ait été utilisé. En effet à certains endroits où la craie est très compacte, des outils en silex ont vraisemblablement été employés. Même si nous n'avons retrouvé qu'un petit nombre de pics miniers, certaines des traces laissées dans de la craie très compacte montrent l'utilisation d'outils de section plus large qu'une pointe en bois de cerf (St. 185 par exemple). Mais dans la majorité des cas le bois de cerf est la matière la mieux adaptée que ce soit dans le descellement des blocs ou dalles de craie ou dans les étapes de déchaussement des rognons de silex.

La série des "Orlets" est très fournie, puisqu'elle compte 567 pièces. Elle est donc tout à fait comparable à celle des grandes minières européennes comme Grimes Graves par exemple (CLUTTONBROCK J.,1982). On y trouve tant des ébauches que des chutes de débitage (traces de fabrication sur place) que des outils utilisés. Parmi ces derniers il existe une typologie variée composée de :

- pics à poignée intégrée (merrain et andouiller) ; - des pics à poignée ajoutée (avec parfois traces d'emmanchement) ;

- outils perforés ;

- burins (au sens propre du terme) ;

- descelleurs de rognons ;

- percuteurs.

La série s'avère très intéressante en comparaison des trois autres déjà étudiées sur l'A5. Au total on dispose maintenant de 800 outils environ, distribués sur les sites du "Grand Bois Marot", des "Orlets", du "Buisson Gendre" à Pâlis et du "Revers de Brossard" à Serbonnes. Tant au niveau des supports que des techniques de fabrication que de la typologie des pièces, la série des "Orlets" constitue un hybride entre les séries les mieux documentées du "Grand Bois Marot" (SIDERA I., 1991 b et sous presse) et de Serbonnes dans l'Yonne (SIDERA 1991 a). Elle représente vraisemblablement une chronologie étagée entre le Néolithique moyen et le Néolithique final.

\section{LA PRODUCTION EN SILEX}

Grâce à la conservation exceptionnelle des états de surface de la minière de Villemaur-sur-Vanne "Le Grand Bois Marot" (LABRIFFE P.-A. de et al. 1990, LABRIFFE P.-A. de et THEBALUT D. 1992 et sous presse), l'analyse lithique des amas de taille et des nombreuses ébauches de hache avait permis de mettre au point des méthodes d'étude adaptées à ce type de restes et de produits et ainsi de réunir des renseignements consistants sur les modalités de la transformation en ébauches de hache de la matière extraite (AUGEREAU A., sous presse). La minière des "Orlets" dont il est question dans cet article, présente des structures de surface moins bien préservées et une étude aussi poussée y est impossible. Toutefois, les résultats obtenus au "Grand Bois Marot" auront permis de valider ceux réunis aux "Orlets", plus partiels.

\section{IDENTIFICATION DE LA PRODUCTION}

Comme au "Grand Bois Marot", aux "Orlets", l'intention de production reste majoritairement la hache. On rencontre néanmoins une activité secondaire orientée vers la fabrication de lames et d'éclats, minoritaire. On a aussi pu mettre en évidence une curieuse méthode de débitage d'éclats qui se rapprocherait de la méthode Levallois (une dizaine de nucléus) : les nucléus sont préparés, à partir des bords, par enlèvements centripètes unifaciaux ou bifaciaux. De la préforme ainsi obtenue, on a tiré de deux à cinq éclats. Pour le Néolithique, ce type de chaîne opératoire ne serait connu actuellement qu'à Canneville (Oise), en contexte Chasséen septentrional (HAMARD D., 1987). Les restes de taille, piégés essentiellement dans les têtes de puits, contiennent, comme au "Bois Marot", 
un taux important d'éclats de façonnage de pièces bifaciales, les restes correspondant aux autres chaînes opératoires étant rares et de toute façon difficilement distinguables de ceux issus des phases de dégrossissage des ébauches. Ici, on s'intéressera plus particulièrement à la chaîne opératoire de façonnage des ébauches de hache.

\section{LA COMPOSITION DES RESTES DE TAILLE ET L'ORGANISATION DE LA PRODUCTION}

De même que pour le "Grand Bois Marot", il s'agissait de déterminer si toutes les étapes de la chaîne opératoire de fabrication des ébauches prêtes à polir étaient représentées au sein des restes de taille dans le but de préciser l'organisation spatiale de la production (AUGEREAU A., sous presse) et d'ébaucher des hypothèses sur le dispositif socioéconomique de l'activité minière (LECH J., 1981 et 1982). facts. Les éclats épais à très épais partiellement corticaux, également taillés par percussion dure, forment de $43 \%$ (st. 136) à $65 \%$ (st. 36) du poids des éclats aux Orlets alors qu'ils n'en représentaient que $32 \%$ à $44 \%$ au "Bois Marot". Ceci peut s'expliquer par le fait que, aux" Orlets", un certain pourcentage de ce type d'éclats provient des chaînes opératoires secondaires (débitages de lames et d'éclats), gonflant ainsi les effectifs de cette catégorie. Les catégories d'éclats suivantes sont caractéristiques de la taille bifaciale et peuvent être assignées sans ambigüité à la chaîne opératoire de façonnage des ébauches. Les éclats sans cortex ou portant du cortex en partie distale (phase de mise en forme, par percussion tendre) enregistrent quelques variations avec $5 \%$ du poids des artefacts pour la st. 100 à $15 \%$ pour la st. 136. Mais, étant donnée l'existence, dans cette minière, de chaînes opératoires secondaires, il a fallu isoler les éclats sans cortex ne montrant pas, de manière évidente,

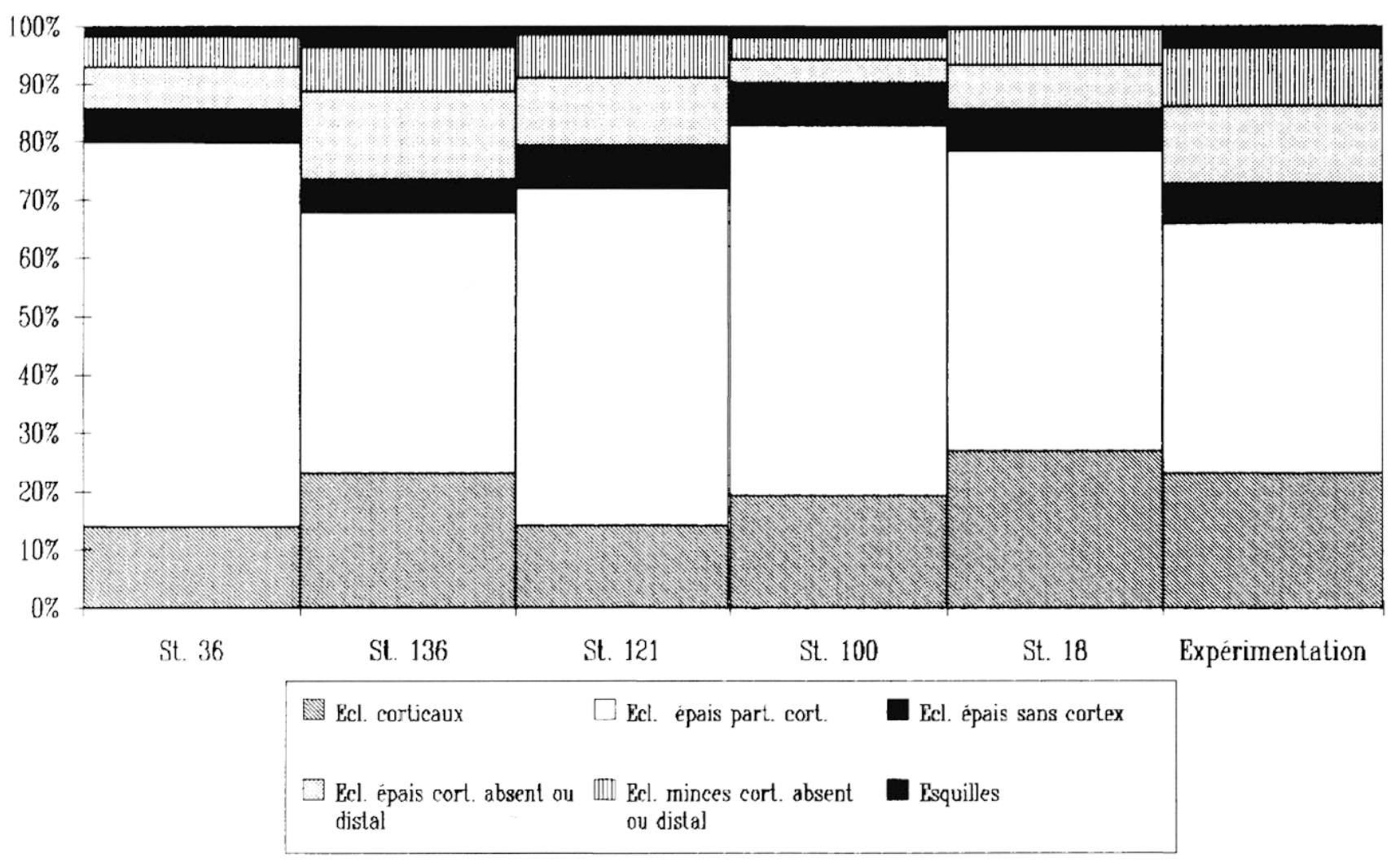

Fig. 10: Graphique de répartition des restes de taille entre structures et par rapport à la taille expérimentale

$\mathrm{D}^{\prime}$ une manière générale la composition des restes de taille (poids en grammes des catégories techniques définies dans AUGEREAU A., sous presse) varie faiblement d'une structure à l'autre et les différentes phases de façonnage des haches, clairement mises en évidence au "Bois Marot" grâce à la conservation exceptionnelle des amas et certifiées par l'unité de la production, se retrouvent aux "Orlets" dans des proportions voisines : la représentation pondérale des éclats corticaux, épais ou très épais, taillés par percussion dure (phase de dégrossissage des blocs, fig. 10) regroupe de $15 \%$ (st. 36 et 121) à $25 \%$ (st. 18) du poids total des arte- les critères de reconnaissance d'éclats de façonnage de hache. Cette distinction n'est pas sans effet sur la représentation des autres catégories d'éclats et notamment les éclats provenant de façon certaine du façonnage de hache. La phase de régularisation (éclats minces - épaisseur inférieure à $5 \mathrm{~mm}$ - cortex absent ou partie distale) est bien homogène avec $5 \%$ (st. 100) à $8 \%$ (st. 136) du poids total des restes aux "Orlets". Pour les mêmes raisons, évoquées au paragraphe précédent, elle est moins abondante qu'au "Bois Marot". Quant au poids d'esquilles de taille bifaciale, il varie d'une structure à l'autre (de 2 à $5 \%$ du poids des éclats), selon le mode de prélèvement sur le terrain des assem- 
blages lithiques (fouille manuelle, récolte des ensembles lors du creusement de la tranchée d'exploration à la pelle mécanique...).

La répartition des restes de taille est cohérente avec celle des produits de la taille expérimentale effectuée par J. Pelegrin (ERA 28 du CNRS, fig. 10) et réalisée sur une dizaine de blocs provenant des deux bancs principaux exploités (bancs 3 et 4). On trouve en effet environ $20 \%$ du poids des restes expérimentaux parmi les éclats corticaux, $41 \%$ parmi les éclats épais partiellement corticaux, $13 \%$ parmi les éclats épais sans cortex ou portant du cortex sur la partie distale (la phase de mise en forme des ébauches) et $8 \%$ parmi les éclats minces sans cortex ou portant du cortex sur la partie distale (régularisation).

Par comparaison avec les données de la taille expérimentale, on peut avancer que la chaîne opératoire de façonnage des haches est complète et que la fabrication des ébauches prêtes à polir s'est effectuée sur le site d'extraction, du moins pour les structures traitées, comme au "Grand Bois Marot".

\section{DEGRES DE TECHNICITE ET PREPARATION DES ARETES DE L'EBAUCHE AU STADE DE LA REGULARISATION}

L'approche expérimentale a un autre avantage : elle permet de replacer le matériel archéologique par rapport à un "étalon" expérimental édifié d'après l'observation archéologique et une grande fréquence de pratique (PELEGRIN J., 1991 a, AUGEREAU A., sous presse). De cette manière, un degré de savoir-faire peut être déterminé, pouvant être corrélé, d'après l'étude ethno-archéologique de Roux et Pelegrin (1989), à différents niveaux de spécialisation techno-économiques.

L'observation des restes de taille des étapes à risques du façonnage, comme la phase de régularisation préparant au polissage, est susceptible $\mathrm{d}^{\prime}$ apporter les résuiltats les plus significatifs sur le niveau de savoir-faire. Ce sont les degrés de prépa- ration des bords de l'ébauche, lisibles sur les talons des éclats provenant de cette phase, qui ont fournit les données les plus révélatrices d'un procédé de régularisation peu soigné : seulement $15 \%$ à $25 \%$ de talons facettés (et donc attestant d'une préparation des bords de l'ébauche) parmi les éclats archéologiques, contre près de $40 \%$ pour les éclats issus de l'expérimentation.

Cette particularité n'a sans doute pas été sans conséquence sur la forme finale des ébauches, moins régulières et moins équilibrées que des pièces dont la phase de régularisation est soigneusement menée. Les phases de polissage ont du en être rendues de ce fait plus pénibles et plus longues et on pourrait penser que les tailleurs des "Orlets", comme ceux du "Grand Bois Marot", avaient une faible fréquence de pratique.

\section{CAUSES D'ABANDON DES EBAUCHES ET "PIECES DEVIANTES"}

La conservation des amas de surface du "Grand Bois Marot" avait permis, par comparaison avec la taille expérimentale, de proposer une estimation des temps de travail et du nombre d'ébauches abandonnées par rapport à celles emportées et donc conformes aux standards des lames prêtes à polir. Par opposition avec la production d'un tailleur ayant une grande fréquence de pratique comme J. Pelegrin, le nombre de pièces défectueuses du "Grand Bois Marot" semblait assez important (de 15 à $50 \%$ de ébauches ont été jugées non satisfaisantes, AUGEREAU A., sous presse). On en avait conclu que ces observations confortaient l'hypothèse de tailleurs non spécialisés d'un point de vue technique, exerçant occasionnellement à Villemaur, déjà décelable dans l'étude des degrés de préparation des enlèvements à risque. Une telle analyse est impossible aux "Orlets" mais on peut approcher indirectement cette question par l'étude des causes d'abandon des ébauches.

Le tableau II montre que plus de la moitié des ébauches de stade 2 (ébauches au stade de la mise

\begin{tabular}{|l|c|c|c|c|}
\hline \multicolumn{1}{|c|}{ CAUSES D'ABANDON } & \multicolumn{2}{c|}{ ORLETS } & \multicolumn{2}{c|}{ BOIS MAROT } \\
\hline \multicolumn{1}{|c|}{} & $\mathrm{N}$ & $\%$ & $\mathrm{~N}$ & $\%$ \\
\hline CAUSES NON PREVISIBLES: & & & & \\
- Failles & 16 & 16,5 & 10 & 18,2 \\
\hline CAUSES PREVISIBLES ET EVITABLES: & & & & \\
- Enlèvements profonds & 15 & 15,5 & 6 & 10,9 \\
- Enlèvements scalariformes & 34 & 35 & 14 & 25,5 \\
- Cassure & 23 & 23,7 & 14 & 25,5 \\
- Bloc inadapté & 5 & 5,2 & 8 & 14,5 \\
\hline INDETERMINEES & 4 & 4,1 & 3 & 5,4 \\
\hline TOTAL DES CAUSES & 97 & 100 & 55 & 100 \\
\hline
\end{tabular}

Tableau II : causes d'abandon des ébauches sur les sites du "Grand Bois Marot" et des "Orlets". 
en forme) et de stade 3 (ébauches au stade de la régularisation) récoltées lors de la fouille des "Orlets"et du "Grand Bois Marot" ont été rejetées à la suite d'évènements prévisibles et évitables pour un tailleur pratiquant souvent (PELEGRIN J., 1991 b) et que l'on peut qualifier de maladresses : enlèvements scalariformes ou trop profonds, choix de blocs inadaptés, cassures dues à un mauvais maintien de l'ébauche lors de certains enlèvements à risque.

A priori, ces résultats appuieraient l'idée de tailleurs peu performants, pratiquant exceptionnellement, et d'une absence de spécialisation technoéconomique de la production d'ébauches de hache prêtes à polir aux "Orlets". Cependant, il faut attirer l'attention sur une notion échafaudée par Chauchat (1991), celle de "pièces déviantes" c'est à dire non conformes aux normes de production recherchées car présentant des caractéristiques aberrantes.

Il semblerait que la plupart des ébauches trouvées aux "Orlets" soient des pièces déviantes (fig. 11). Mais il est possible de déterminer différents degrés de "déviance" dont les extrêmes seraient représentés par des ébauches franchement "déviantes" s'opposant à celles semblant les plus proches des standards de fabrication. Ces dernières sont rares : on en conterait seulement une dizaine sur les 160 pièces répertoriées. Les autres ébauches montrent des caractères extravagants. Du point de vue du choix du support d'une part, la grande majorité de ces pièces a été taillée dans des rebuts d'extraction (petits blocs oblong à plats ou de petites plaquettes de moins bonne qualité que les rognons recherchés en priorité). Du point de vue métrique d'autre part, une partie de ces ébauches exhibe des rapports lon- gueur/largeur, longueur/épaisseur ou largeur/épaisseur anormaux par comparaison avec les pièces a priori non déviantes, les rendant non fonctionnelles. Enfin, la plupart d'entre elles cumule plusieurs causes d'abandon prévisibles (réfléchissements répétés, enlèvements trop profonds, mauvais choix de blocs...).

La combinaison de l'ensemble de ces particularités rend insuffisante l'hypothèse d'un manque de pratique de l'ensemble des tailleurs ayant oeuvré aux "Orlets". En fait, tout se passe comme si les portions de matière première jugées inadaptées à la fabrication de haches fonctionnelles aient été abandonnées à des tailleurs débutants. Sans mettre en avant l'hypothèse d'un apprentissage organisé des artisans sur le site, on pourrait imaginer que les plus jeunes, et donc les tailleurs les moins expérimentés, récupéraient les déchets de l'extraction pour s'exercer à la taille. Pour préciser cette hypothèse, l'établissement d'une échelle de degrés de savoir-faire plus détaillée sur la base d'un support expérimental serait d'un grand secours. Ces observations pourraient mener à l'idée que les restes de taille présents sur le site seraient uniquement issus de ces tailleurs débutants et que tous les matériaux extraits propres à la production auraient été emportés et taillés ailleurs. Toutefois, si cette hypothèse était vraie, la distribution en catégories techniques des éclats divergerait plus nettement de celle de la taille expérimentale. D'autre part, l'abondance relative des éclats de régularisation n'est pas en rapport avec le nombre d'ébauches au stade de la régularisation, qui se chiffre à quelques individus (seulement 11 sur 160 ébauches de tous

Fig. 11 : ébauche de hache présentant des caractères "déviants" (Dessin A. Augereau).
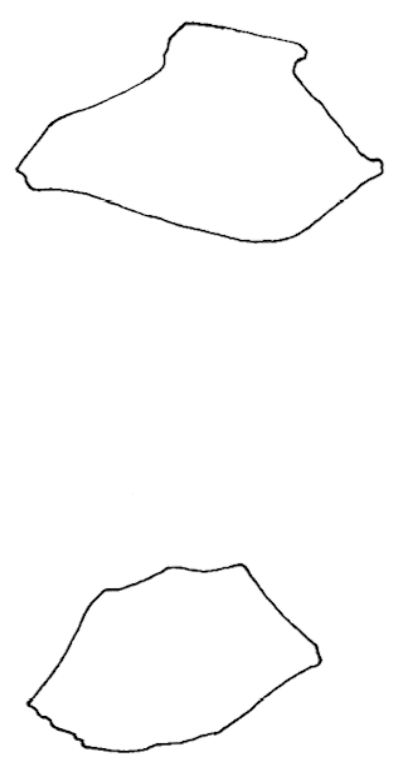
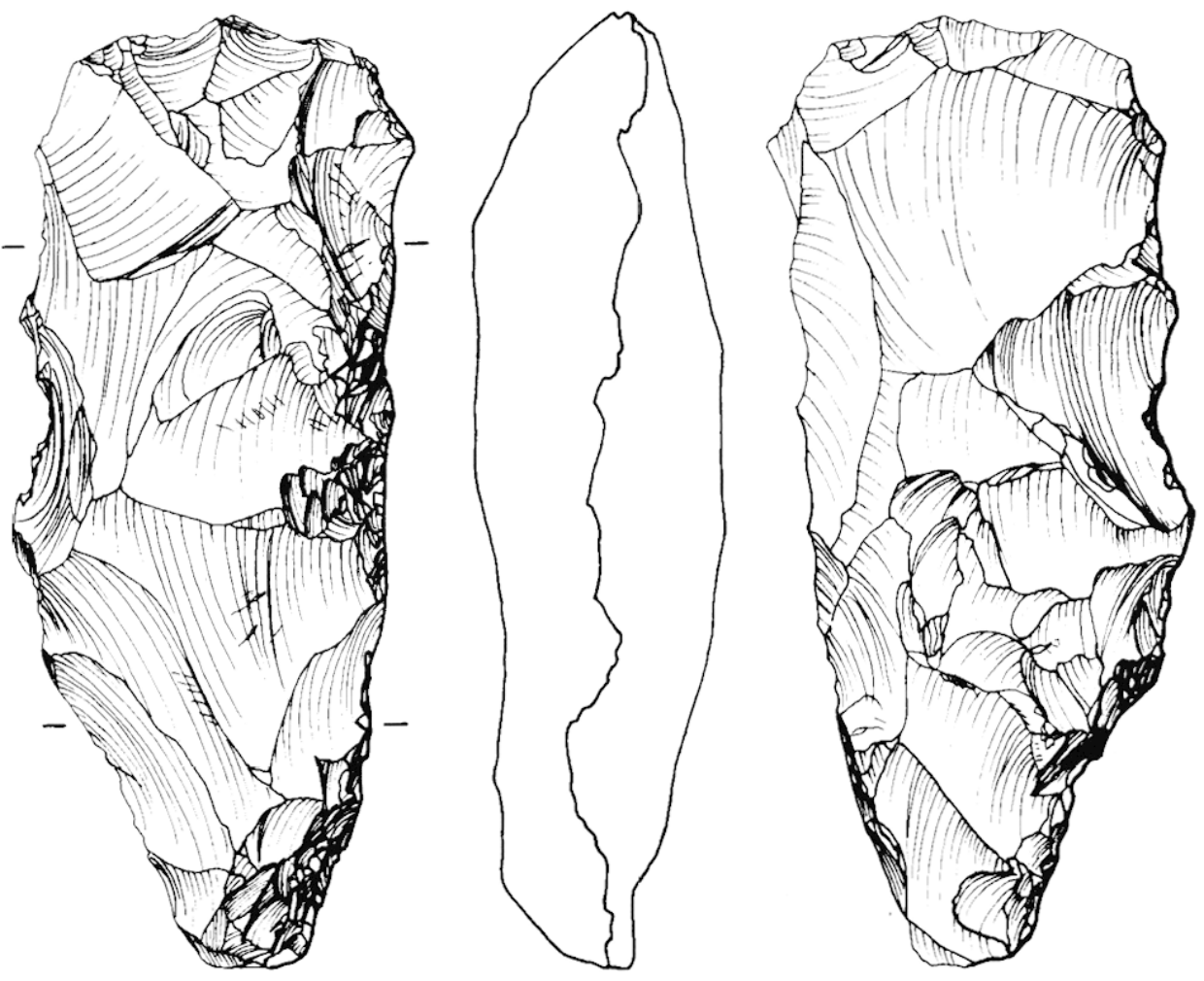
stades). Enfin, il existe quelques pièces non déviantes, délaissées pour des causes techniques moins graves, et qui, à l'évidence, ont été taillées sur le site.

Ainsi, aux "Orlets", dans l'état actuel des investigations, les résultats convergent vers l'idée d'une absence de spécialisation techno-économique de la production d'ébauches de hache, en faveur de laquelle les données réunies au "Grand Bois Marot" militaient. Le degré de savoir-faire relativement bas, comme en témoigne l'étude des modes de préparation des bords de l'ébauche, et le fait que l'ensemble de la chaîne opératoire, sauf le polissage, semble s'être déroulée à proximité immédiate du lieu d'extraction, indiqueraient une division du travail faible. Par ailleurs, la destination probablement locale des produits miniers (abondantes collections de surface comprenant des lames de hache polies) et l'abondance des sites d'extraction dans les vallées voisines occupées au Néolithique iraient en faveur d'une exploitation casuelle des gisements de matière première, satisfaisant à des besoins familiaux, voire villageois. Toutefois, on doit s'interroger sur les faits que les rognons affleurant en abondance ne suffisent plus et que les populations éprouvent la nécessité de pratiquer l'extraction minière dans le but de fabriquer des haches. Ceci peut apparaître néanmoins comme une forme de spécialisation, non pas socio-économique mais simplement technique, peut être en rapport avec des contraintes techniques et économiques.

\section{ELEMENTS REMARQUABLES}

Bien que marginaux, il nous faut signaler la découverte de quelques éléments plutôt rares en contexte minier. Comme toujours très peu de céramique puisque nous n'avons récupéré qu'une demi-douzaine de tessons, ce qui proportionnellement au cubage remué est particulièrement faible. Toutefois, dans un des puits test (St. 18) nous avons retrouvé un vase entier (LABRIFFE P.-A. de et al., 1992). C'est un des premiers trouvé dans une structure d'extraction (GUILLAUME et al. 1987). Il était situé juste sous les couches constituant le remplissage terminal du puits qui dans cette structure semble naturel. Vu son emplacement, son état de conservation, ainsi que la stratigraphie de ce puits, il peut être considéré comme contemporain du fonctionnement de cette structure.

Il s'agit d'un vase qui rappelle les vases tulipiformes d'affinité Michelsberg. Mais l'aspect ubiquiste de cette forme, le caractère isolé de cette trouvaille au sein de la minière et la localisation du site aux confins des aires culturelles actuellement définies (N.M.B, Noyen, Michelsberg, Chasséen), de même que le manque de comparaisons locales, rendent difficile une attribution chrono-culturelle précise et donnent à cette découverte un caractère un peu anecdotique.

Au moment de la réalisation des tranchées profondes, nous avons également retrouvé une sépulture d'enfant. Le corps avait été déposé dans un puits à environ $80 \mathrm{~cm}$ de la surfacc. Il reposait sur le côté droit, jambes et bras repliés, la tête orientée au sud-sud-ouest. Pour tout mobilier funéraire il disposait d'un collier constitué de perles en bois de cerf imitant des craches de cerf, de canines (perforées ou non) et d'une perle tubulaire en os d'oiseau.

\section{DATATION}

Nous ne disposons pas encore des datations radiocarbones. Mais plusieurs éléments plaident en faveur d'une longue occupation du gisement. L'extrême densité des structures et leurs nombreux recoupements laissent supposer qu'un temps suffisamment conséquent s'est écoulé pour que les mineurs perdent la connaissance des structures excavées antérieurement et que celles-ci ne laissent plus aucune trace dans le paysage.

Le vase est à placer dans un Néolithique moyen qui reste à préciser au niveau chrono-culturel. Les techniques de fabrication de l'outillage en bois de cerf, elles, semblent faire appel à des pratiques que l'on retrouve au Néolithique moyen et au Néolithique final. L'occupation sans doute relativement étalée dans le temps, ne semble pas débuter avant le Néolithique moyen et pourrait perdurer jusqu'au Néolithique final.

\section{CONCLUSION}

Il reste encore de très nombreuses questions en suspens. Mais la mise en perspective des trois minières précédemment fouillées sur le tracé de l'A5 (Serbonnes "Le Revers de Brossard", Palis "le Buisson Gendre" et Villemaur-sur-Vanne "le Grand Bois Marot") avec celle de Villemaur-sur Vanne "les Orlets" devrait bientôt être achevée. Ces travaux devraient déboucher sur la publication des différents résultats concernant ces sites au sein d'un volume de la collection Archéologie et Grands travaux des DAF. Chacun des gisements sera caractérisé sous différents aspects (gitologie, acquisition de la matière première, production ...), puis on essaiera de préciser les liens et relations pouvant unir les composantes du complexe minier, enfin nous tenterons d'apprécier l'impact et le rayonnement de cet ensemble dans le contexte du Néolithique régional. Dans les années à venir il est également envisagé de poursuivre des recherches sur le phénomène minier dans le Pays d'Othe dans le cadre d'opérations programmées de recherche. 


\section{BIBLIOGRAPHIE}

AUGEREAU A. (1991 a) - Villemaur-sur-Vanne "Le Grand Bois Marot"; étude de la production en silex rapport de sauvetage programmé. Coordination Archéologique des Autoroutes A5-A160, 1991. 31 p. 14 fig. (Autoroute A5; Melun-Troyes ; études archéologiques, rapport dactylographié).

AUGEREAU A. (1991 b) - "Etude des ateliers de fabrication de haches de la minière de Villemaursur-Vanne (Aube) : objectifs et méthodologie", dans : Les mines de silex au Néolithique en Europe occidentale. Pré-actes, Table ronde internationale, Vesoul (Haute-Saône), 18-19 Octobre 1991. 1 p.

AUGERAU A. (1991 c) - "Nature de la production lithique, objectifs et méthodologie pour le traitement de grandes séries de matériel", dans : $L a$ Bourgogne entre les bassins rhénan, rhodanien et parisien, carrefour ou frontière. Résumé des communications. XVIIIème Colloque interrégional sur le Néolithique, Dijon, Octobre 1991, p. 49.

AUGEREAU A. (sous presse) - "Les ateliers de fabrication de haches de la minière du Grand Bois Marot à Villemaur-sur-Vanne (Aube)", dans : Les mines de silex au Néolithique en Europe occidentale. Actes de la table ronde internationale, Vesoul (Haute-Saône), 18-19 Octobre 1991.

BOSTYN F. dir., LANCHON Y. dir. (1992) - Jablines Le Haut-Chateau (Seine-et-Marne), une minière de silex au Néolithique, Paris, Maison des Sciences de l'Homme, 1992, 246 p., (Archéologie et Grands travaux, Document d'Archéologie Française $\mathrm{N}^{\circ} 35$ ).

CLUTTON-BROCK J., (1982) - "Neolithic antler picks from Grimes Graves, Norfolk, Durrington Walls, Wiltshire : a biometrical analysis", dans : Excavations at Grimes Graves Norfolk 1972-1976, fasc. 1, 44 p., 12 fig.

CHAUCHAT C. (1991) - "L'approche technologique dans une étude régionale : le Paijanien de la cìte du Pérou", dans : 25 ans d'études technologiques en Préhistoire, XIème Rencontres Internationales d'Archéologie et d'Histoire d'Antibes, Antibes 1990, Ed. APDCA, Juan-les-Pins, 1991. p. 263-174.

GUILLAUME C., LIPINSKI P., MASSON A. (1987) - Les mines de silex néolithiques de la Meuse dans le contexte européen, Editions des Musées de la Meuse, 1987, $70 \mathrm{p}$.

HAMARD D. (1987) - Le site chasséen de Canneville (Oise). Etude du matériel lithique et céramique. Documents d'Archéologie Française $\mathrm{N}^{\circ} 11$, Ed. de la Maison des Sciences de l'Homme, Paris 1987. $171 \mathrm{p}$.
HASCOET J., LABRIFFE P-A. de, MENDOZA Y ALMEIDA M. (1993) - "Le site minier de Serbonnes "Le Revers de Brossard" ; premiers résultats et perspectives (Yonne, Autoroute A5)", dans : Le Néolithique au quotidien, Actes du XVIème colloque interrégionnal sur le Néolithique, Paris 5-6 Novembre 1989. Documents d'Archéologie Française $\mathrm{N}^{\circ} 39$, Paris : Maison des Sciences de l'Homme, p. 177-189.

HASCOET J., MENDOZA Y ALMEIDA M. (1991 a) - "Mines de silex et bois de cerf, l'exemple de Serbonnes le "Revers de Brossard" (Yonne); Introduction", Revue Archéologique de l'Est et $d u$ Centre-Est, t.42, fasc. 1, fasc. semestriel 159, Janvier-Juin 1991. Paris, Ed. du C.N.R.S., 1991, p. 63-64.

HASCOET J., MENDOZA Y ALMEIDA M. (1991 b) - Pâlis "Le Buisson Gendre ; minière néolithique ; rapport de diagnostic ; proposition de sauvetage programmé. Coordination Archéologique des Autoroutes A5-A160, 1991, 24 p., 17 fig. (Autoroute A5; MelunTroyes; études archéologiques, rapport dactylographié).

HASCOET J., MENDOZA Y ALMEIDA M. (1991 c) - Pâlis "Le Buisson Gendre", 10277001 AP, minière néolithique. Rapport de Sauvetage Programmé. Coordination Archéologique des Autoroutes A5A160, 1991, 2 t., 47 p., 127 fig. (Autoroute A5 Melun-Troyes, études archéologiques, rapport dactylographié).

JOURDAIN D. (1991) - "Le mégalithisme dans l'Aube", dans : Actes du $15^{\circ} \grave{e} m e$ colloque Interrégionnal sur le Néolithique, Châlons-sur-Marne 22-23 Octobre 1988, A.R.P.E.P.P, 1991, p. 137-147.

LABRIFFE P.-A. de (1991) - Villemaur-sur-Vanne "le Grand Bois Marot" minière de silex néolithique ; rapport préliminaire, demande de prolongation de sauvetage programmé. Coordination Archéologique des Autoroutes A5-A160, 1991. 7 p., pl et fig. (Autoroute A5, Melun-Troyes, études archéologiques, rapport dactylographié).

LABRIFFE P.-A. de (1992) - Villemaur-sur-Vanne "Les Orlets", site d'extraction de silex ; rapport de diagnostic, proposition de sauvetage programmé, Coordination Archéologique des Autoroutes A5A160, 1991, 17 p., 19 fig. (Autoroute A5; MelunTroyes, études archéologiques, rapport dactylographié).

LABRIFFE P.-A. de, AUGEREAU A., SIDERA I., THEBAULT D. (1991) - "Studying technical systems in the Pays-d'Othe mining complex (Aube, France), The example of Villemaur-sur-Vanne "Le 
Grand Bois Marot" : extraction, antler tools, flint production", dans : Pre-print VI International Flint Symposium, Madrid, 5-6 Octobre 1991. 3 p., 2 fig.

LABRIFFE P.-A. de, DELEPINE J., AUGEREAU A. (1990) - Villemaur-sur-Vanne "Le Grand Bois Marot"; 10415407 (numérotation provisoire A5); site d'extraction de silex. Rapport de diagnostic approfondi, proposition de sauvetage programmé, Coordination Archéologique des Autoroutes A5-A160, 1990, 23 p., 26 fig. (Autoroute A5, Melun-Troyes, études archéologiques, rapport dactylographié).

LABRIFFE P.-A. de, THEBAULT D. (1991 a) "Autoroute A5 et minières de silex", dans : Les mines de silex au Néolithique en Europe occidental, Pré-actes Table ronde internationale, Vesoul (Haute-Saône), 18-19 Octobre 1991, 2 p.

LABRIFFE P.-A. de, THEBAULT D. (1991 b) - "Le complexe minier du Pays d'Othe : l'exemple de Villemaur-sur-Vanne "Le Grand Bois Marot", dans : La Bourgogne entre les bassins rhénan, rhodanien et parisien, carrefour ou frontière, résumé des communications. XVIIIème Colloque interrégional sur le Néolithique, Dijon, 25-27 Octobre 1991,1p.

LABRIFFE P.-A. de, Thébault D. (1992) - Villemaursur-Vanne "Le Grand Bois Marot" : site d'extraction, atelier de fabrication de haches; rapport de Sauvetage Programmé. Coordination Archéologique des Autoroutes A5-A160, 1991, 44 p., 50 fig. (Autoroute A5, Melun-Troyes, études archéologiques, rapport dactylographié).

LABRIFFE P.-A. de, THEBAULT D. (sous presse) "Mines de silex et grands travaux, 1 'autoroute A5 et les sites d'extraction du Pays-d'Othe", dans : Les mines de silex au Néolithique en Europe occidentale. Actes de la table ronde internationale, Vesoul (Haute-Saône), 18-19 Octobre 1991, 17 p., 7 fig.

LAPORTRE L. (1992) - "Adaptation du logiciel Excel pour la réalisation d'images tri-dimensionnelles", dans : Jablines Le Haut Chateau (Seine-etMarne), une minière de silex au Néolithique, annexe 2. Paris : Maison des Sciences de l'Homme, 1992, p. 230-232, (Archéologie et Grands travaux, Document d'Archéologie Française $\mathrm{N}^{\circ} 35$ ).

LECH J. (1981) - Flint mining among the early farming communities in Poland", dans : Actes $d u$ IIIème symposium international du silex, Maastricht, mai 1979, Staringia $n^{\circ} 6$, Ed. Engelen, Sittard, 1981, p. $39-45$.

LECH J. (1982) - "Flint mining among the early farming communities in Central Europe. Part II : the basis of research into flint workshops", dans : Przeglad Archeologiczny, vol. 30, Varsovie, 1982, p. 47-80.
MENDOZA Y ALMEIDA M., HASCOET J. (1990) Serbonnes Le Revers de Brossard, minière néolithique ; rapport de sauvetage programmé, Coordination Archéologique des Autoroutes A5-A160, 1990, 104 p., 83 fig., pl. (Autoroute A5, Melun-Troyes, études archéologiques, rapport dactylographié).

MUSEE DES BEAUX-ARTS et D'ARCHEOLOGIE DE TROYES (1990) - "A la découverte des mégalithes de l'Aube, dolmens-menhirs et polissoirs", Catalogue de l'exposition Le mégalithisme dans l'Aube, (21 Avril au 4 juin 90), Edition des Musées de Troyes et de l'ARPEPP, 1990, 103 p., photos, dessins, bibliographie.

PELEGRIN J. (1991 a) - "Aspects de démarche expérimentale en technologie lithique" dans : 25 ans d'études technologiques en Préhistoire, XIème Rencontres Internationales d'Archéologie et d'Histoire d'Antibes, Antibes 1990, Ed. APDCA, Juan-les-Pins, 1991. p. 57-63.

PELEGRIN J. (1991 b) - "Réflexions méthodologiques sur l'étude de séries lithiques en contexte $\mathrm{d}^{\prime}$ ateliers ou de mines", dans : Les minières néolithiques en Europe occidentale, Résumés des communications de la table ronde de Vesoul, octobre 1991, $1 \mathrm{p}$.

ROUX V., PELEGRIN J. (1989) - “Taille des perles et spécialisation artisanale. Enquête ethno-archéologique dans le Gujarat", dans : Techniques et Cultures, t. 14, fasc. juillet-décembre, Ed. de la Maison des Sciences de l'Homme, Paris, 1989. pp. 23-49.

SIDERA I. (1991 a) - "Mines de silex et bois de cerf, l'exemple de Serbonnes le "Revers de Brossard" (Yonne)", dans : Revue Archéologique de l'Est et du Centre-Est, t.42, fasc. 1, fasc. semestriel 159, Janvier-Juin 1991, Paris, Ed. du C.N.R.S., 1991, p. 63-91.

SIDERA I. (1991 b) - Outillage d'extraction, les éléments en bois de cerf du Grand Bois Marot à Villemaur, rapport de sauvetage programmé, Coordination Archéologique des Autoroutes A5-A160, 1991, 47 p., 36 fig. (Autoroute A5, Melun-Troyes, études archéologiques, rapport dactylographié).

SIDERA I. (1991 c) - "Relations minières habitats : un problème de méthode. Le potentiel des artefacts osseux", dans : Les mines de silex au Néolithique en Europe occidentale, Pré-actes, Table ronde internationale, Vesoul (Haute-Saône), 18-19 Octobre 1991. 2 p., 1 pl.

SIDERA I. (1993) - Villemaur-sur-Vane "Les Orlets", outillage d'extraction, les éléments en bois de cerf, Rapport d'étude, Coordination Archéologique des Autoroutes A5-A160, 1993, 20 p. fig. (Autoroute 
A5, Melun-Troyes, études archéologiques, rapport dactylographié)

SIDERA I. (sous presse) - "Relations minières habitats : un problème de méthode. Le potentiel des artefacts osseux", dans: Les mines de silex au Néolithique en Europe occidentale, Actes de la table ronde internationale, Vesoul (Haute-Saône), 18-19 Octobre 1991, 22 p.

SOULIER P. (1971)- L'extraction du silex en Europe occidentale (Allemagne, Angleterre, Belgique, France). 161 p. (mémoire de maîtrise de l'Université de Paris I, dactylographié, 1971). 